\title{
On the relationship between knowledge and memory for pictures: Evidence from the study of patients with semantic dementia and Alzheimer's disease
}

\author{
KIM S. GRAHAM, ${ }^{1,2}$ JAMES T. BECKER, ${ }^{3}$ AND JOHN R. HODGES ${ }^{1,2}$ \\ ${ }^{1}$ University Neurology Unit, Addenbrooke's Hospital, Cambridge, CB2 2QQ, U.K. \\ ${ }^{2}$ MRC Applied Psychology Unit, 15 Chaucer Road, Cambridge, CB2 2EF, U.K. \\ ${ }^{3}$ Neuropsychology Research Program, Departments of Psychiatry and Neurology, \\ University of Pittsburgh Medical Center, Pittsburgh, PA 15213 \\ (Received November 7, 1996; Revised April 7, 1997; Accepted April 29, 1997)
}

\begin{abstract}
Current views of long-term memory presume that both the hippocampal complex and the neocortex play interactive, but separate, roles in the storage of memories. While the neocortex is considered the eventual and permanent store for our memories, the encoding of recently experienced events is thought to be initially dependent upon the hippocampus and closely related structures. Neuropsychological studies have demonstrated that damage to the medial temporal lobe results in a retrograde amnesia extending back in time, with better preservation of older memories. The converse pattern has been shown in patients with semantic dementia, who have focal atrophy of the inferolateral temporal neocortex, but relative sparing of the hippocampal complex (Graham \& Hodges, 1997). Here we demonstrate that such patients can show relatively preserved new learning on a forced-choice recognition memory test (based on real and chimeric animals), while patients in the early amnestic phase of Alzheimer's disease show severely impaired learning on the same test. This result provides support for the view that new learning is primarily dependent upon the hippocampus and related structures. (JINS, 1997, 3, 534-544.)
\end{abstract}

Keywords: Episodic memory, Hippocampus, Temporal neocortex, Long-term memory storage

\section{INTRODUCTION}

\section{Current Views of Long-Term Memory Storage: Neuropsychological Evidence}

Over the past 20 years there has been considerable debate concerning the fractionation of long-term memory. One particularly influential model is that first proposed by Tulving (1972, 1983) who hypothesized the division of long-term memory into episodic and semantic memory. The term episodic memory applies to our store of personally based memories, and involves conscious recollection of the specific temporal-spatial setting of a previously experienced event. For example, in an episodic memory test, the participant might have to recall or recognize words they had seen in a

Reprint requests to: Kim Graham, University Neurology Unit, Box 165, Addenbrooke's Hospital, Cambridge, CB2 2QQ, U.K. E-mail: Kim. Graham@mrc-apu.cam.ac.uk. previous study list, or tell the experimenter what they were doing when they heard that Margaret Thatcher had resigned. Semantic memory, on the other hand, applies to our knowledge of the world, including vocabulary, concepts and facts: Information that can be retrieved without recalling when and where it was learned (e.g., a patient may have to verify that Margaret Thatcher was a Conservative Prime Minister or define the meanings of words (such as "politician," "rhinoceros," "table," etc.). Tulving (1972) suggested that episodic and semantic memory were both psychologically and neurologically distinct systems, and that this dichotomy reflected the way the brain processed and stored information.

It was initially hoped that research with amnesic patients would support Tulving's (1972) hypothesis, as it was proposed that the deficits found in amnesics could be explained in terms of impaired episodic but preserved semantic memory (Kinsbourne \& Wood, 1975; Parkin, 1982). Research over the last decade, however, has suggested that this simple dichotomy is probably incorrect. For example, am- 
nesic patients have equal difficulty in acquiring new facts and in learning about new events (Shimamura \& Squire, 1987). Amnesic Korsakoff's patients also show a striking impairment in semantic knowledge about the world (as tested by their ability to recognize famous scenes and faces and recent vocabulary, when that knowledge is related to events that occurred many years before, as well as since, the onset of the amnesia; e.g., Kopelman, 1989; 1993; Hodges, 1994; Verfaellie et al., 1995). Comparisons of episodic and semantic memory from the distant past and more recent past (i.e., after the onset of amnesia) also demonstrate that amnesic patients can recall episodic memories from early life (ZolaMorgan et al., 1983). These studies have cast some doubt upon the view that amnesia is simply a selective impairment of episodic memory.

While the results from studies with classic amnesic patients suggest that episodic and semantic memory may not be functionally or neurologically separate, clinical observations from patients with semantic dementia appear to offer support for such a separation. The term semantic dementia, first coined by Snowden et al. (1989), has recently been widely adopted to describe patients with progressive fluent aphasia in whom the breakdown in language processing reflects an underlying loss of semantic memory (Hodges et al., 1992, 1994; Breedin et al., 1994; Snowden et al., 1994). On tests of picture naming (and in spontaneous speech) the patients have severe word finding difficulties, yet show preservation of the phonological and syntactic aspects of language. In addition, there is loss of both word and object meaning. Nonverbal problem-solving, basic perceptual and visuospatial abilities, and working memory are notably unaffected, even at relatively advanced stages of the disease (Hodges et al., 1994).

With respect to episodic memory, the patients seem well oriented and, within the literature, there are anecdotal reports of relatively preserved recall of recent events. Clinical observations of our patients confirm these accounts: One of our patients reported to her husband a phone call 3 hours earlier, but could not remember the name of the person who had rung; on another occasion, a patient was able to recall who had tested him, where he was tested, and the type of experiments he had received over the past week in hospital. In addition, it is not uncommon for patients with semantic dementia to produce relatively detailed (if anomic) autobiographical accounts of events from their recent past in spontaneous speech (Hodges et al., 1992; Graham \& Hodges, 1997). These detailed autobiographical memories are in striking contrast to the profound loss of semantic memory that affects the patients' recognition of common objects and influences their everyday functioning and communication (Schwartz et al., 1979; Snowden et al., 1989, 1994; Diesfeldt, 1992; Hodges et al., 1992, 1994).

Although there have been few detailed studies of episodic memory in these patients, Diesfeldt $(1987,1992)$ tested new learning in a single case using a forced choice recognition memory task: The patient was $100 \%$ correct on recognition of five pictures after a delay of $10 \mathrm{~min}$, and, in addition, remembered where the experimenter had hidden three objects after a delay of approximately $1 \mathrm{hr}$. This was in striking contrast to the patient's profound loss of semantic memory, impaired single-word comprehension and impoverished general knowledge.

Some researchers, however, have proposed that patients with progressive loss of semantic memory have impaired episodic memory; for example, Horner (1990) points out that the patients included in Warrington's (1975) paper on selective impairment of semantic memory were impaired on two subtests from the Wechsler Memory Scale (recall of a short story and reproduction of visual designs from immediate memory; Wechsler, 1987). Furthermore, on two tests of long-term memory (a forced-choice recognition memory test for words, faces and paintings, and a test of retention of words) the patients were almost as impaired as global amnesics (except for their recognition of paintings). Warrington (1975) writes, however,

Yet these patients were not at all like amnesic patients with a global amnesia for all on-going events. Unlike an amnesic patient they (all three patients) were well-oriented in time and place; their conversation was not so repetitive and they were able to refer forwards and backwards to detailed events of importance in their lives. (p. 650)

It seems, therefore, that we still do not fully understand the nature of long-term memory and that the neuropsychological data from patients with amnesia and patients with semantic memory loss is contradictory. Tulving's (1972, 1983) dichotomy between episodic and semantic memory is almost certainly simplistic, since it assumes that the acquisition, storage, and retrieval of these two putative categories of information depends upon separate systems. This view cannot realistically be correct: If amnesic patients have difficulty acquiring both new semantic facts and new events after the onset of the amnesia, surely this indicates that both types of information may be acquired via the same system. Furthermore, the fact that some patients with amnesia can recall events from their childhood, but not from their recent past seems to imply that not all events (i.e., new and old) are dependent upon the same neuroanatomical structure or system. Moreover, there is now convincing evidence that patients may selectively lose (or retain) certain domains of long-term memory. For instance, autobiographical memory may be severely impaired, yet knowledge of famous persons from the same period is preserved (Hodges \& McCarthy, 1993; Evans et al., 1996) and vice versa (Evans et al., 1995).

More recent, data-driven, approaches to the study of longterm memory have been based, therefore, on a consideration of both neuropsychological and neuroanatomical research. Models, especially computer-based formulations of memory processes that emphasize the temporal dimension of long-term memory, the nature of the material stored, and the type of reconstructive processes required to recall incidents and facts offer more comprehensive hypotheses against which to test neuropsychological data (Alvarez \& 
Squire, 1994; McClelland et al., 1995; Squire \& Alvarez, 1995; Murre, 1996).

\section{Long-Term Memory Storage: A Computational Perspective}

In current computational models of long-term memory storage, permanent representations are established via interactions between two different neuroanatomical regions: the hippocampal complex and the neocortex. It is believed that the hippocampal complex is crucial for the initial, temporary encoding of an experienced event, while the neocortex is the permanent repository for long-term memories. There are a number of computational instantiations of this theory: Murre (1996), and also Alvarez \& Squire (1994), suggest that new (temporary) episodic memories are initially encoded through connections between higher-order cortical areas and the hippocampal complex. With repeated rehearsal, corticocortical connections develop in the neocortex, allowing the representation and recall of the memory to become independent of the hippocampal complex. By contrast, McClelland and colleagues (1995) propose that recently experienced memories are actually stored in the hippocampus. Over time, the hippocampus-dependent memories are interleaved with memories already permanently represented in the neocortex. Damage to specialized regions of neocortex could impair specific domains of knowledge.

These time-based views of memory, in which memories are "transferred" within the brain, provide an explanation for much of the amnesic literature. For example, as both new factual information and new events would be encoded via the same process (see McClelland et al., 1995), it is no surprise that amnesic patients have equal difficulty encoding new semantic and episodic memories. Furthermore, the models also account for the fact that amnesic patients show a pattern of retrograde amnesia: Damage to structures in the medial temporal lobe will result in the loss of all memories entirely dependent on the hippocampus. More distant memories encoded in the neocortex will be unaffected.

With respect to the disorder of semantic dementia, there is neuroradiological and neuroanatomical evidence to support the view that the hippocampal complex is relatively spared, in contrast to severe, circumscribed atrophy of the inferolateral temporal neocortex (Hodges et al., 1992; Harasty et al., 1996; Hodges \& Patterson, 1996). It is predicted therefore, that both new learning and recall of memories from the recent past should be unimpaired in such patients (the opposite pattern to that seen in amnesia). By contrast, the permanent representations of experiences from the more distant past (e.g., childhood) together with the factual database of knowledge that constitutes semantic memory, will be disrupted and lost since it is assumed that both depend upon neocortical regions that are severely atrophic. It has already been established that one prediction from the computational models is correct: Patients with semantic dementia remember events from the recent past substantially better than events from the distant past (see Graham \& Hodges, 1997). As mentioned previously, however, the second prediction, that patients should show relatively normal performance on tests of nonverbal new learning, ${ }^{1}$ is controversial.

The aim of this study was to investigate the integrity of new learning in patients with semantic dementia using an experiment based on an object decision task (Humphreys et al., 1988). We predicted that the patients would be impaired on the object decision test, either because the task requires access to visually based semantic knowledge (as suggested by Chertkow et al., 1992) or alternatively because interactions with semantic memory are necessary for the integrity of stored structural descriptions (see Patterson \& Hodges, 1992; Graham et al., 1994; Hodges et al., 1994, for further discussion of this issue). By contrast, we predicted that the patients would be unimpaired on a forcedchoice recognition memory test based on the same items. A group of patients in the early amnesic phase of Alzheimer's disease (in whom the pathological processes are presumed to involve the hippocampal complex preferentially) were also tested in this study: It was predicted that they would show the opposite pattern of performance to the patients with semantic dementia (i.e., normal performance on the object decision test, impaired learning on the object decision recognition memory test).

\section{Research Participants}

A total of 19 individuals participated in the study: 4 patients in the early amnesic stage of presumed Alzheimer's disease ( 2 male and 2 female), 5 patients with semantic dementia ( 3 male and 2 female), and 10 age-matched (to the semantic dementia patients) controls (5 male and 5 female). The mean age for the patients with presumed AD was 72.5 years $(S D=5.51)$, for the patients with semantic dementia, 59.8 years $(S D=2.2)$ and for the controls, 59.3 years $(S D=4.1)$. Average years of education were $9(S D=0)$, $13(S D=4.19)$ and $10.5(S D=8)$ for the patients with Alzheimer's disease (AD), the semantic dementia patients, and the control participants, respectively.

The amnesic AD patients presented to the Memory Clinic at Addenbrooke's Hospital, Cambridge with an informant confirmed history of an insidiously progressive anterograde memory disorder, which was interfering with everyday functioning. The patients with semantic dementia also presented to the Memory Clinic, complaining of difficulties with word production (especially for the names of people, places, and words that were previously familiar to them). Spouses confirmed the anomia and also noted difficulties with comprehension of word meaning. All 9 patients have been studied longitudinally, at 6-month intervals, over the

\footnotetext{
${ }^{1}$ It should be noted that patients with semantic dementia perform poorly on tests of verbal new learning (e.g., the Logical Memory subtest from the Wechsler Memory Scale, 1987; see Table 1). It is suspected that this impairment reflects the problems the patients have with language production and comprehension, rather than a specific deficit in new learning.
} 
past 3 years, during which time detailed neuropsychological testing has confirmed the original clinical observations.

The amnesic AD patients have shown a progressive decline in new learning of both verbal and nonverbal material. At presentation in 1993 they showed no other cognitive difficulties, but over time they have developed mild semantic memory (as measured using category fluency, in which the subject generates as many exemplars from a category, such as animals, as possible) and/or visuospatial deficits (copying of the Rey Complex Figure: Osterrieth, 1944; and Judgment of Line Orientation: Benton et al., 1983).

Table 1 shows the performance of the 4 AD patients on a battery of neuropsychological tests. In all 4 cases, the testing was given approximately 3 to 6 months after the experiment described in this paper. On the tests of semantic memory (Hodges Semantic Battery: Hodges et al., 1992; Hodges \& Patterson, 1995), two of the AD patients (R.B. and H.M.) show impaired performance: Both were impaired on a picture naming test, in which they had to name 48 relatively high-frequency black-and-white line drawings, a naming-to-descriptions test, in which the patient has to name an item from a description (e.g., "a small green animal which leaps around ponds"), and on a category fluency test. These 2 patients (R.B. and H.M.) were also mildly impaired on the picture version of the Pyramid and Palmtrees Test (Howard \& Patterson, 1992) of associative semantic knowledge, in which the participant decides which of two pictures-words goes best with a target (e.g., target $=$ windmill, related item $=$ tulip, foil $=$ daffodil $)$. The other 2 patients (I.H. and S.C.) performed well on most of the tests of semantic memory, apart from category fluency, which seems to be a sensitive marker of early semantic memory impairment (Hodges \& Patterson, 1995). The 4 AD patients were markedly impaired on all tests of episodic memory: recall of the Rey Complex Figure; Warrington's Recognition Memory Test (RMT; Warrington, 1984); and the delayed logical memory component from the Wechsler Memory ScaleRevised (Wechsler, 1987). I.H., although impaired on the words component of the RMT (Warrington, 1984), showed normal performance on the recognition of unfamiliar faces. The AD patients have also started to show impairment of visuospatial and perceptual abilities: Although only S.C. was impaired on Benton's Judgment of Line Orientation Test, all 4 patients produced poor copies of the Rey Figure.

It should be noted that patients with a history of closed head injury, excessive alcohol intake or with risk factors for cerebrovascular disease were excluded from this group. A full battery of investigations, including CT and/or MRI has failed to reveal any alternative cause for the patients' memory loss. Studies of similar patients have suggested that such an isolated amnesic prodrome may present for as much as a decade prior to the development of a full-blown dementia syndrome (Weintraub \& Mesulam, 1993; Lucchelli et al., 1994; Tanabe et al., 1994).

Table 1. A comparison of the performance of the 4 amnesic patients with presumed early Alzheimer's disease (and controls; see Hodges \& Patterson, 1995) on a range of neuropsychological tests

\begin{tabular}{|c|c|c|c|c|c|}
\hline \multirow[b]{2}{*}{ Tests } & \multirow{2}{*}{$\begin{array}{c}\text { Controls }(N=24) \\
M(S D)\end{array}$} & \multicolumn{4}{|c|}{ Patient } \\
\hline & & I.H. & R.B. & S.C. & H.M. \\
\hline \multicolumn{6}{|l|}{ Language } \\
\hline NART $(n=50)$ & $39 \quad(6.2)$ & 35 & 26 & 26 & 18 \\
\hline TROG $(n=80)$ & $78.8 \quad(1.8)$ & 79 & 78 & 76 & 70 \\
\hline \multicolumn{6}{|l|}{ Semantic memory } \\
\hline Naming (48) & $43.6 \quad(2.3)$ & 48 & 22 & 44 & 35 \\
\hline Naming to description (24) & 22.5 (1.3) & 23 & 9 & 21 & 15 \\
\hline Category fluency-living & $58.5 \quad(12.3)$ & 24 & 11 & 26 & 23 \\
\hline Category fluency-manmade & $55.4 \quad(8.6)$ & 24 & 18 & 35 & 18 \\
\hline Word-Picture matching (48) & $47.4 \quad(1.1)$ & 48 & 47 & 45 & 45 \\
\hline PPT ( 3 pictures, $n=52$ ) & $51.2(1.4)$ & 50 & 44 & 48 & 44 \\
\hline \multicolumn{6}{|l|}{ Episodic memory } \\
\hline Recall of Rey figure (36) & $15.25(7.4)$ & 3 & 0 & 2 & 4 \\
\hline WRMT-faces (50) & $44 \quad(3.8)$ & 50 & NT & 35 & 34 \\
\hline WRMT-words (50) & $47 \quad(2.8)$ & 42 & NT & NT & 27 \\
\hline Logical memory-delayed (24) & $8.5 \quad(3.4)$ & 0 & 0 & 0 & 0 \\
\hline \multicolumn{6}{|l|}{ Visuospatial } \\
\hline Rey copy (36) & $34 \quad(2.9)$ & 17.5 & 25 & 12 & 16 \\
\hline Line orientation (30) & $27.4 \quad(4.0)$ & 30 & 30 & 19 & 27 \\
\hline
\end{tabular}

Results are taken from tests given close to the time of the study. Tests (and abbreviations) are as follows: NART = National Adult Reading Test (Nelson, 1982); Rey Figure (Osterrieth, 1944); TROG = Test for the Reception of Grammar (Bishop, 1989); PPT = Pyramid and Palm Trees Test (Howard \& Patterson, 1992); WRMT = Warrington Recognition Memory Tests (Warrington, 1984); Line Orientation (Benton et al., 1983); Logical Memory = subtest from the Wechsler Memory Scale-Revised (Wechsler, 1987); NT $=$ Not tested. 
The 5 patients with semantic dementia have shown progressive difficulty with word production and single-word comprehension. At time of presentation, all 5 showed marked impairment on subtests from the Hodges semantic battery (Hodges et al., 1992; Hodges \& Patterson, 1995). Table 2 shows the degree to which the patients were impaired on the picture naming test, the naming-to-descriptions test and category fluency. On tests of semantic comprehension, such as selection of an item from a number of foils (wordpicture matching) and the Pyramid and Palm Trees test (Howard \& Patterson, 1992), all 5 patients exhibited evidence of knowledge loss (see Table 2). Four of the patients; J.L., G.C., P.S., and A.M., showed moderate to severe loss of semantic memory, which has worsened considerably over time. The 5th patient, F.M., showed only modest impairment on measures of semantic memory when she first presented, and over time, despite a profound and progressive anomia, has only just started to show any significant loss of semantic knowledge (4-5 years after she first presented). Elsewhere, we have described her as a case of progressive pure anomia (see Graham et al., 1995). Although she did not initially fulfill the criteria for semantic dementia, we have included her in this study in spite of her milder semantic memory impairment, so as to provide a spectrum of defi- cits. Like most other reported cases of progressive fluent aphasia, none of the patients with semantic dementia have shown noticeable decline on tests tapping other cognitive domains; for example, all 5 continue to perform well on tests of working memory (digit span), perceptual and visuospatial abilities (e.g., copying the Rey Figure; Osterrieth, 1944), nonverbal problem-solving (e.g., Coloured Matrices; Raven, 1962) and syntactic comprehension (e.g., the Test for the Reception of Grammar; Bishop, 1989).

\section{NEUROANATOMY: MRI}

Coronally oriented MRI scans showed that 3 of the 5 patients with semantic dementia (P.S., G.C., and F.M.) had focal atrophy of the left inferolateral temporal lobe, with relative preservation of the hippocampus and parahippocampal gyrus. The other 2 patients (J.L. and A.M.) had atrophy of parallel regions of both the right and left temporal lobes, with more marked involvement of the right side in J.L., and the left side in A.M. The hippocampal region in both patients looked relatively preserved.

$\mathrm{CT}$ and/or coronally oriented MRI scans of the brains of the amnesic $\mathrm{AD}$ patients revealed selective and asymmetric

Table 2. A comparison of the performance of the 5 patients with semantic dementia (and controls; see Hodges \& Patterson, 1995) on a range of neuropsychological tests

\begin{tabular}{|c|c|c|c|c|c|c|}
\hline \multirow[b]{2}{*}{ Tests } & \multirow{2}{*}{$\begin{array}{c}\text { Controls }(N=24) \\
M(S D)\end{array}$} & \multicolumn{5}{|c|}{ Patient } \\
\hline & & J.L. & G.C. & F.M. & P.S. & A.M. \\
\hline \multicolumn{7}{|l|}{ Language } \\
\hline NART $(n=50)$ & $39 \quad(6.2)$ & 4 & 0 & 4 & NT & 11 \\
\hline TROG $(n=80)$ & $78.8 \quad(1.8)$ & 73 & 73 & 72 & 71 & 75 \\
\hline \multicolumn{7}{|l|}{ Semantic memory } \\
\hline Naming (48) & $43.6 \quad(2.3)$ & 9 & NT & 4 & 5 & 3 \\
\hline Naming to description (24) & $22.5(1.3)$ & 4 & 2 & 3 & 0 & 0 \\
\hline Category fluency-living & $58.5 \quad(12.3)$ & 13 & NT & 4 & 3 & 12 \\
\hline Category fluency-manmade & $55.4(8.6)$ & 12 & NT & 9 & 5 & 6 \\
\hline PALPA word-picture matching (40) & $38.9 \quad(2.2)$ & 29 & 34 & 35 & 27 & 30 \\
\hline Word-picture matching (48) & $47.4 \quad(1.1)$ & 27 & 23 & 44 & 24 & 36 \\
\hline Picture pointing (S \& V, $n=260)$ & NT & 192 & 233 & 250 & 192 & NT \\
\hline PPT ( 3 pictures, $n=52$ ) & $51.2(1.4)$ & 36 & 44 & 46 & 37 & 39 \\
\hline \multicolumn{7}{|l|}{ Episodic memory } \\
\hline Recall of Rey figure (36) & $15.25(7.4)$ & 8 & 12 & 8 & 9.5 & 12.5 \\
\hline WRMT-faces (50) & $44 \quad(3.8)$ & 22 & 30 & 46 & 35 & 30 \\
\hline WRMT-words (50) & $47 \quad(2.8)$ & 32 & 23 & 34 & NT & NT \\
\hline Logical memory-delayed (24) & 8.5 (3.4) & 0 & NT & 0.75 & 0 & 2.5 \\
\hline \multicolumn{7}{|l|}{ Visuospatial-problem-solving } \\
\hline Rey copy (36) & $(2.9)$ & 34 & 36 & 30 & 35 & 36 \\
\hline Line orientation (30) & $27.4 \quad(4.0)$ & 27 & NT & 21 & 23 & 30 \\
\hline Coloured matrices (36) & NT & NT & 34 & 22 & 36 & NT \\
\hline
\end{tabular}

Results are taken from tests given close to the time of the study. It should be noted that some of the tests were not given to G.C. He was, however, poor at picture naming tests [on the Boston Naming Test (Kaplan et al., 1983) he scored 6 correct] and category fluency (for animals he produced 6 items). Like the other patients, he showed normal performance on visuospatial tests. For tests (and abbreviations) see legend to Table 1 (also PALPA Word-picture matching (Kay et al., 1992); Coloured Matrices (Raven, 1962); and S \& V = Snodgrass \& Vanderwart (1980). 
hippocampal atrophy, with relatively little involvement of the temporal lobes.

\section{METHOD}

\section{Materials}

The same 32 items were used in both the semantic (object decision) and the episodic (forced-choice object decision recognition memory) tests. These were selected from the object decision test designed by Humphreys et al. (1988). Fourteen of these items were real animals, and 18 were nonreal, chimeric animals (e.g., the body of a dog and a donkey's head; see Figure 1 for examples). In addition, 32 novel items (14 real and 18 nonreal), selected from the original object decision test, were used in the recognition memory component of this experiment.

\section{Procedure}

The two tests were as follows:

\section{Object decision}

The participant viewed each of the 32 line drawings of real and nonreal animals singly in random order and was asked to indicate whether they thought the picture was real or nonreal by responding "yes," or "no," respectively.

\section{Object decision recognition memory test}

Five min after the object decision test, the participant was presented with 32 pairs of line drawings. One of the line drawings in every pair had been seen previously by the participant in the object decision test. The other member of the pair was a novel item. The pairs were matched; that is, a real familiar picture was always paired with a real novel one, and the same was true for the nonreal pictures. The
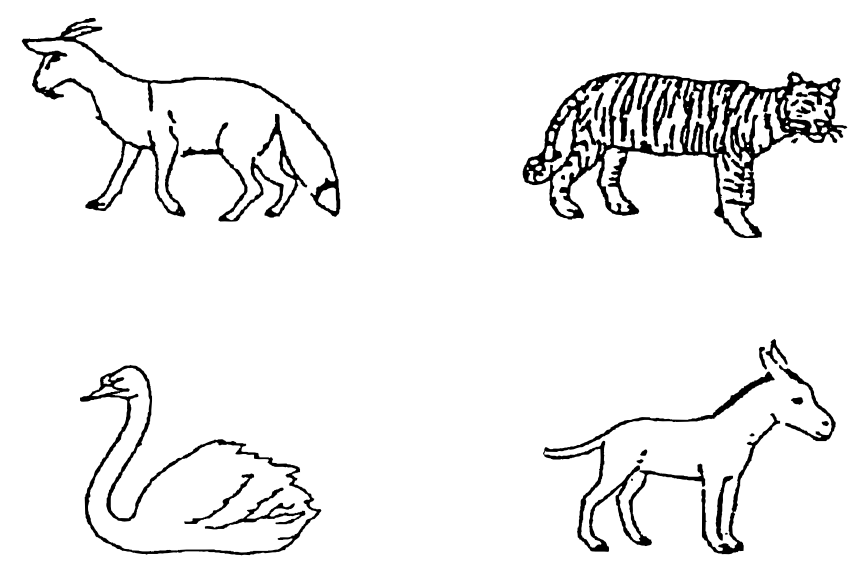

Fig. 1. Examples from the object decision test (Humphreys et al., 1988). participant was asked to indicate, by pointing, which picture in the pair they had seen in the object decision test.

\section{RESULTS}

The results from each version of the object decision test (i.e., the semantic and episodic components) were analyzed separately using one-way factorial ANOVAs. The one-way ANOVA revealed a significant main effect of group $[F(2,16)=24.7, p<.001]$; post-hoc Scheffé pairwise comparisons indicated that the patients with semantic dementia were significantly worse on the object decision test than the other two subject groups [AD vs. SD: mean difference $(\delta)=4.0, p<.005$; controls $v s$. SD: $\delta=6.6, p<.001]$. By contrast, there was no difference, although there was a trend towards significance, between the AD patients and the controls $(\delta=-2.5, p=.07)$. Figure $2 \mathrm{a}$, which illustrates the mean performance of the three participant groups on the object decision test, confirms the results from the ANOVA and post-hoc analyses.

The one-way ANOVA for the object decision recognition memory test, like that for the object decision, revealed a significant main effect of group $[F(2,16)=20.2, p<.001]$. Post-hoc Scheffé pairwise comparisons, however, revealed a converse pattern to that described above: The patients with semantic dementia were as good at the recognition memory task as the controls ( $\delta=1.2, p=.46)$, while the amnesic Alzheimer patients showed significantly poorer performance than both the control subjects and the semantic dementia patients (AD vs. controls: $\delta=-10.7, p<.001$; AD vs. SD: $\delta=-9.5, p<.001)$. Figure $2 \mathrm{~b}$ demonstrates the difficulty the amnesic Alzheimer's disease patients have with the recognition memory test, compared to the other two participant groups.

To allow a comparison of the performance of the individual patients with reference to that of the 10 control subjects, $z$-score conversions (for both the object decision and the recognition component) were performed. Table 3 demonstrates that, although all 5 patients with semantic dementia obtained scores that were at least 2 standard deviations outside the control mean in the object decision task, none of the patients performed outside this range in the object decision recognition memory test. It should be noted that F.M. was only marginally more impaired than the control participants on the object decision test, a fact that is consistent with her milder semantic memory impairment.

The amnesic patients were all at least 2 standard deviations outside the control mean on the object decision recognition memory test, although 1 patient (I.H.) was only mildly impaired. On the object decision test, 2 of the patients (R.B. and S.C.) showed evidence of some difficulty judging whether the animals were real or not. It is not clear, at least from this study, whether this reflects a semantic or a visuospatial deficit. The other 2 amnesic Alzheimer patients showed no impairment on the object decision task, although it should be noted that none of the patients achieved a score that was near the top range of the control group. 


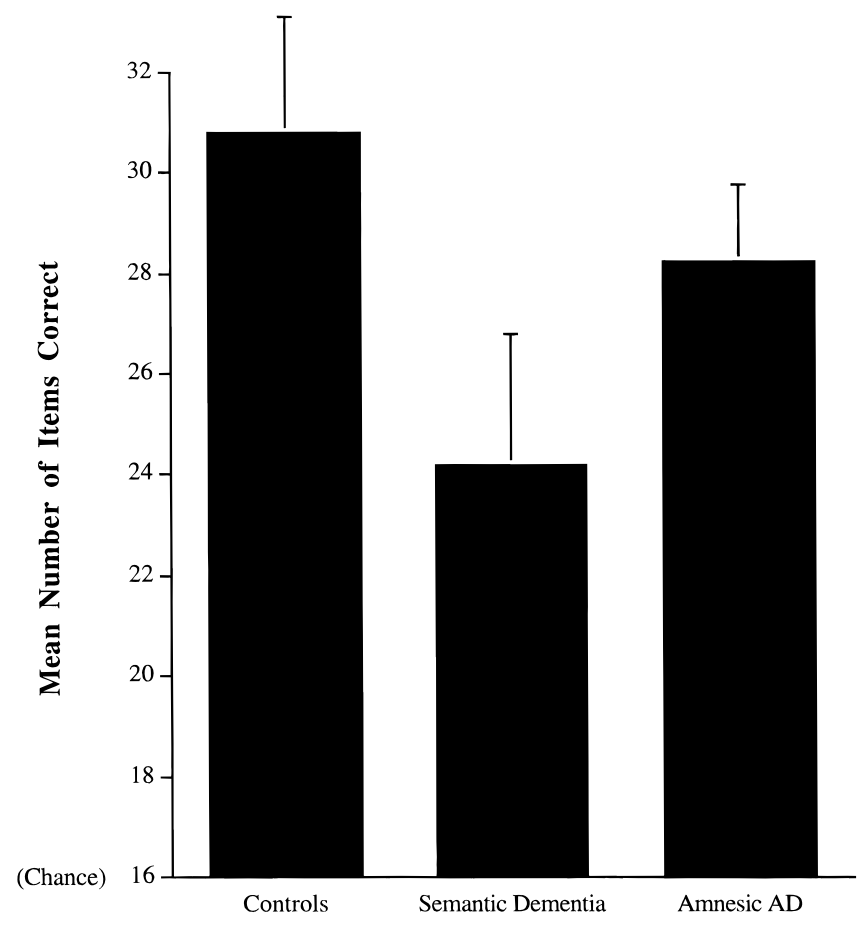

Object Decision

Fig. 2a. Mean number of items correct on the object decision test for the patients with semantic dementia, the amnestic patients with presumed Alzheimer's disease and the control participants. Standard deviations are represented as bars. The horizontal axis starts at chance (number correct $=16$ ).

\section{DISCUSSION}

Our hypothesis that patients with semantic dementia, at least at a relatively early stage in the disease, would show preservation of nonverbal new learning, was based on clinical observations of their behavior, and the fact that their MRI scans showed relative preservation of the neuroanatomical structures (the hippocampus, parahippocampal gyrus, etc.) thought to be crucial for encoding new memories. In this study, all the semantic dementia patients performed poorly (to varying degrees) on the object decision test, yet showed relatively preserved performance on the two-alternative forced-choice recognition memory test based on the same items. This result demonstrates that the patients can correctly identify which items they saw in the object decision test, even though they were sometimes unable to say whether these pictures were true or false representations of the animals. By contrast, the amnesic patients with presumed early Alzheimer's disease performed poorly on the forced-choice recognition memory test. Two of these patients showed performance within 2 standard deviations of the control participants on the object decision test, while the other 2 showed a mild impairment.

Our results support those found by Diesfeldt $(1987,1992)$ in a pilot study involving a single semantic dementia patient who remembered all items in a four-alternative forced-

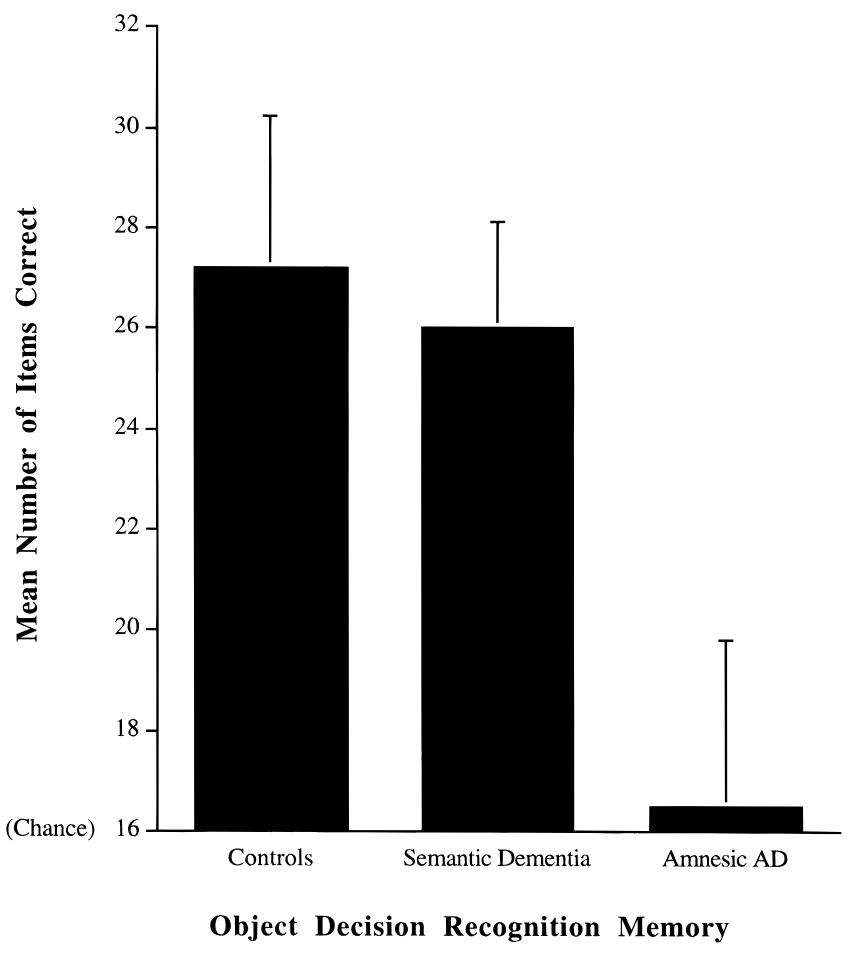

Fig. 2b. Mean number of items correct on the object decision recognition memory test for the patients with semantic dementia, the amnestic patients with presumed Alzheimer's disease and the control participants. Standard deviations are represented as bars. The horizontal axis starts at chance (number correct $=16$ ).

choice recognition test (after a delay of $10 \mathrm{~min}$ ). By contrast, Warrington's (1975) patients were as poor as global amnesics when they were given two-alternative forced-choice recognition memory tests of words and faces, although they

Table 3. The performance of each patient on the object decision and object decision recognition memory tests with respect to the mean of the control subjects (measured using $z$ scores)

\begin{tabular}{|c|c|c|}
\hline \multirow[b]{2}{*}{ Patient } & \multicolumn{2}{|c|}{$z$-scores } \\
\hline & $\begin{array}{c}\text { Object } \\
\text { decision }\end{array}$ & $\begin{array}{l}\text { Object decision } \\
\text { recognition } \\
\text { memory }\end{array}$ \\
\hline \multicolumn{3}{|c|}{ Semantic dementia } \\
\hline J.L. & -7.92 & -.72 \\
\hline G.C. & -3.90 & -.39 \\
\hline F.M. & -2.28 & -.72 \\
\hline P.S. & -7.15 & -1.37 \\
\hline A.M. & -4.7 & .26 \\
\hline \multicolumn{3}{|c|}{ Amnesic Alzheimer's disease } \\
\hline I.H. & -.65 & -2.03 \\
\hline R.B. & -3.09 & -3.67 \\
\hline S.C. & -3.09 & -4.66 \\
\hline H.M. & -1.46 & -3.67 \\
\hline
\end{tabular}


performed within the control range when asked to recognize paintings they had been shown previously. Warrington proposed that some aspect of the paintings (she suggested the color) was meaningful to her patients, which allowed them to score as well as control participants. Since our object decision test involved black-and-white pictures, this hypothesis cannot account for our patients' success.

The most likely explanation for the pattern of results across the various studies is that patients with semantic dementia show normal performance on tests of recognition memory when verbal encoding strategies are inappropriate or unavailable. This may happen in tests where all pictures are similar (landscapes) or where there are similar and repeated items from the same semantic category (object decision). When attempting to remember whether one has seen an item (e.g., a face or a word) in a previous study episode, it is possible to use verbally and/or semantically based strategies (often without conscious awareness) to support recognition. For example, remembering a face because the person resembles your next door neighbor, or someone famous, such as British film actors, for example, John Cleese and Denholm Elliott (see the faces component of the RMT). Words are even more amenable to semantic "scaffolding": first, words already have a meaning; and second, it is possible to create connections between items to aid retention. For example, the first six words presented in the RMT are treat, dive, rush, rest, fall, and bark. A rest could be a treat, dive and fall, both involve dropping, and someone could be rushing because they have been barked at. Patients with semantic dementia are certainly going to be disadvantaged in these types of tests (especially word-based tests), and will therefore process material more shallowly than normal individuals.

There is tentative evidence in support of this hypothesis: Patients with semantic dementia show poorer forced-choice recognition memory on either words or faces depending on which temporal lobe (the left or right) has the greater atrophy. For example, F.M. (see also G.C.), who has more atrophy on the left, scored $46 / 50$ and $34 / 50$ on the faces and words versions of the RMT (Warrington, 1984), respectively; this pattern was reversed in J.L., who had predominantly right-sided atrophy (see Table 2). A patient described by Evans et al. (1995), who had a progressive prosopagnosia with selective atrophy to the right temporal neocortex, showed a similar pattern on the RMT: She was profoundly impaired when asked to remember the unfamiliar faces, yet showed normal performance on the words version and on a test in which she had to remember 50 unfamiliar buildings.

It is important to note that we are not claiming that the semantic dementia patients show entirely normal episodic memory performance. The object decision recognition test used in this study is not a very sensitive index of episodic memory: Only a relatively small number of items $(N=32)$ were used, and the time delay between the object decision test and the recognition memory task was quite short (5 min). Any generalization from this experiment to other circumstances should be circumspect. It is clear that we have at present a limited understanding of the conditions influ- encing the performance of patients with semantic dementia on tests of episodic memory. It is hoped that further studies (for instance, manipulations of the degree of visual and semantic similarity between items used in recognition memory tests) will help clarify this controversial issue.

A further explanation for the pattern of performance seen in patients with semantic dementia is that recognition memory can be supported by familiarity based processes (Schacter, 1987). It is important, therefore, that experiments be undertaken that systematically manipulate the degree of perceptual familiarity between to-be remembered stimuli and targets in the recognition memory test. We suspect that patients with semantic dementia might have greater difficulty with a recognition memory test in which the two items, although the same (e.g., two pianos), vary both with respect to orientation and as many perceptual features (i.e., grand $v s$. upright piano) as possible. Results that confirm this hypothesis would suggest that the "normal" recognition memory performance sometimes seen in semantic dementia may reflect support from intact perceptual processing.

The fact that the patients were able to recall seeing items that they have previously failed to classify appropriately as real or nonreal clearly suggests that patients with degraded knowledge are able to, at least temporarily, retain the memory of novel or partially familiar stimuli. In this study it was not possible to compare performance on individual test items (i.e., those that were "known" or "unknown" to the patients) with performance on the recognition memory test. It seems likely, however, that such an experimental comparison would not reveal a direct one-to-one relationship between what a patient knows about an item, and whether they can remember seeing it in a previous test session.

Other researchers have reached a different conclusion, proposing that episodic memory cannot be intact in patients with loss of semantic memory. Dalla Barba et al. (1996) investigated whether intact episodic memory (as measured using nonverbal recognition memory) could be demonstrated in aphasic (etiology not specified) patients with varying degrees of semantic memory impairment. Three types of pictorial triads were presented to the patients (prior to the recognition memory test): One comprised three semantically unrelated items, and two comprised three semantically related items. In Experiment 1, the patients were presented with 12 semantically unrelated triads one after each other, and given no further instructions. This was followed by Experiment 2, in which the patients were presented with 12 semantically related triads. As before, the patients were given no instructions. In the final experiment (3), 12 (new) semantically related triads were presented to the participants and, unlike Experiments 1 and 2, the participants had to choose which of two items was more related to the target (a test of semantic association). Immediately after presentation of the three series of 12 triads, patients were given a yes-no recognition memory test consisting of the 36 previously studied items.

Dalla Barba et al. noted two results: (1) the aphasic patients, as a group, were poor on the semantic judgment task 
(Experiment 3), and showed poor performance on the recognition memory task (which tested memory for all 36 triads); (2) when the aphasic patients were split into two groups based on their performance on the semantic association task (Experiment 3), the patients who showed poor performance on the semantic association test were worse on the recognition memory task. On the basis of these results Dalla Barba et al. wrote:

The point of this study was to show that when some kind of semantic knowledge about an item is lost, that same item can no longer be recognised in an episodic memory task. This is what the present study shows. (p. 365)

For a number of reasons, we find this a curious interpretation. First, to demonstrate, beyond reasonable doubt, that loss of semantic knowledge about an item leads to an inability to recognize that item in an episodic memory task, it is surely necessary to do direct item-by-item comparisons. Dalla Barba and colleagues did not perform such analyses. Secondly, their results show that episodic memory benefits when the initial encoding stresses semantic processing, not that episodic memory cannot exist without semantic memory. This effect, where semantic encoding produces better retention than nonsemantic processing, is well established (see Craik \& Tulving, 1975) and as mentioned earlier, patients with semantic dementia (like Dalla Barba et al.'s aphasic patients) seem to be influenced both by the material to be learned and the initial encoding processes (the availability of verbally based semantic strategies).

In summary, neither Dalla Barba et al.'s study, nor ours, determine definitively whether a patient can remember seeing an item when they have lost semantic knowledge about that item. Our study does, however, address one of Dalla Barba et al.'s criticisms of the previous literature: They argue that evidence supporting the view that episodic memory is intact in semantic dementia stems only from clinical observations. This is no longer accurate: First, we (Graham \& Hodges, 1997) have demonstrated that patients with semantic dementia are able to retrieve current autobiographical memories; and second, none of the 5 patients tested here showed significant impairment on the nonverbal recognition memory test. It is also helpful to note the relatively good performances seen in all 5 patients when asked to recall the Rey Complex Figure after a delay (Osterrieth, 1944; see Table 2).

The results from the experiment are in keeping with the computational models of long-term memory discussed in the Introduction: It seems that the patients can remember the material from the object decision test over a short period of time, a task that is presumably dependent upon the integrity of the hippocampus. Such learning does not depend upon the complete integrity of the long-term representations required to decide whether an item is real or not. It remains to be established how long such temporary representations can exist; for example, Murre (1996) has suggested that patients with semantic dementia may show abnormal forgetting of newly learned information over time.
At this moment, however, there have been no studies to investigate this possibility.

In summary, this study provides further evidence in support of the view that the neuroanatomical substrate crucial for new learning is distinct from that involved in the representation and recall of semantic knowledge and old autobiographical memories. This result has important implications for patients who have similar, nonprogressive, semantic memory impairments. If, as in semantic dementia patients, the hippocampal system is relatively preserved, it may be possible to devise strategies to help such patients relearn, and maintain by rehearsal, semantic knowledge that has been lost. It is important to note, however, that new learning depends on the connectivity of the hippocampal links with activated areas of the neocortex. The hippocampus may be able to compensate, on a temporary basis, when there is partial breakdown of the neocortical representations, but cannot work in isolation. We postulate that, over time, patients with semantic dementia will inevitably show impoverished nonverbal new learning. Likewise, patients with severe posttraumatic destruction of vital neocortical regions, even in the presence of an intact hippocampus, would show impaired learning.

\section{ACKNOWLEDGMENTS}

We would like to thank Karalyn Patterson for advice and discussions, and two anonymous reviewers for their comments on the paper. We are also grateful to all the patients and control participants for their time and patience. This research was supported by a Wellcome grant to, J.R.H and K.S.G. J.T.B, received support from a Research Scientist Development Award (K02-MH0177) and the Alzheimer's Disease Research Center (AG05133).

\section{REFERENCES}

Alvarez, P. \& Squire, L.R. (1994). Memory consolidation and the medial temporal lobe: A simple network model. Proceedings of the National Academy of Science, 91, 7041-7045.

Benton, A.L., Hamsher, K.deS., Varney, N.R., \& Spreen, O. (1983). Contributions to neuropsychological assessment. New York: Oxford University Press.

Bishop, D.V.M. (1989). Test for the Reception of Grammar (2nd ed.). Manchester, U.K.: Department of Psychology, University of Manchester.

Breedin, S.D., Saffran, E.M., \& Coslett, H.B. (1994). Reversal of the concreteness effect in a patient with semantic dementia. Cognitive Neuropsychology, 11, 617-660.

Chertkow, H., Bub, D., \& Caplan, D. (1992). Constraining theories of semantic memory processing: Evidence from dementia. Cognitive Neuropsychology, 9, 327-365.

Craik, F.I.M. \& Tulving, E. (1975). Depth of processing and the retention of words in episodic memory. Journal of Experimental Psychology: General, 104, 268-294.

Dalla Barba, G., Frasson, E., Montovan, M.C., Gallo, A., \& Denes, G. (1996). Semantic and episodic memory in aphasia. Neuropsychologia, 34, 361-367.

Diesfeldt, H.F.A. (1987). Psychologisch onderzoek van psychogeriatrische patienten volgens de methode van Cahn en Diesfeldt 
[Psychological examination of psychogeriatric patients using the method of Cahn and Diesfeldt]. Laren, The Netherlands: Stichting Verpleeghuizen.

Diesfeldt, H.F.A. (1992). Impaired and preserved semantic memory functions in dementia. In L. Backman (Ed.), Memory functioning in dementia (pp. 227-263). Amsterdam: Elsevier Science.

Evans, J.J., Breen, E.K., Antoun, N., \& Hodges, J.R. (1996). Focal retrograde amnesia for autobiographical events following cerebral vasculitis: A connectionist account. Neurocase, 2, 1-11.

Evans, J.J., Heggs, A.J., Antoun, N., \& Hodges, J.R. (1995). Progressive prosopagnosia associated with selective right temporal atrophy: A new syndrome? Brain, 118, 1-13.

Graham, K.S., Hodges, J.R., \& Patterson, K.E. (1994). The relationship between comprehension and oral reading in progressive fluent aphasia. Neuropsychologia, 32, 299-316.

Graham, K.S. \& Hodges, J.R. (1997). Differentiating the roles of the hippocampal system and the neocortex in long-term memory storage: Evidence from the study of semantic dementia and Alzheimer's disease. Neuropsychology, 11, 77-89.

Graham, K., Patterson, K., \& Hodges, J.R. (1995). Progressive pure anomia: Insufficient activation of phonology by meaning. Neurocase, 1, 25-38.

Harasty, J.A., Halliday, G.M., Code, C., \& Brooks, W.S. (1996). Quantification of cortical atrophy in a case of progressive fluent aphasia. Brain, 119, 181-190.

Hodges, J.R. (1994). Retrograde amnesia. In A. Baddeley, B. Wilson, \& F. Watts (Eds.), Handbook of memory disorders (pp. 81-107) Chichester, U.K.: John Wiley \& Sons.

Hodges, J.R. \& McCarthy, R.A. (1993). Autobiographical amnesia resulting from bilateral paramedian thalamic infarction: A case study in cognitive neurobiology. Brain, 116, 921-940.

Hodges, J.R. \& Patterson, K. (1995). Is semantic memory consistently impaired early in the course of Alzheimer's disease? Neuroanatomical and diagnostic implications. Neuropsychologia, 33, 441-459.

Hodges, J.R. \& Patterson, K. (1996). Non-fluent progressive aphasia and semantic dementia: a comparative neuropsychological study. Journal of the International Neuropsychological Society, 2, 511-525.

Hodges, J.R., Patterson, K., Oxbury, S., \& Funnell, E. (1992). Semantic dementia: Progressive fluent aphasia with temporal lobe atrophy. Brain, 115, 1783-1806.

Hodges, J.R., Patterson, K., \& Tyler, L.K. (1994). Loss of semantic memory: Implications for the modularity of mind. Cognitive Neuropsychology, 11, 505-542.

Horner, M.D. (1990). Psychobiological evidence for the distinction between episodic and semantic memory. Neuropsychology Review, 1, 281-321.

Howard, D. \& Patterson, K. (1992). Pyramids and Palm Trees: A test of semantic access from pictures and words. Bury St. Edmunds, U.K.: Thames Valley Test Company.

Humphreys, G.W., Riddoch, M.J., \& Quinlan, P.T. (1988). Cascade processes in picture identification. Cognitive Neuropsychology, 5, 67-103.

Kaplan, E.F., Goodglass, H., \& Weintraub, S. (1983). The Boston Naming Test (2nd ed.). Philadelphia: Lea \& Febiger.

Kay, J., Lesser, R., \& Coltheart, M. (1992) Psycholinguistic assessment of language processing in aphasia. Hove, U.K.: Lawrence Erlbaum Associates.

Kinsbourne, M. \& Wood, F. (1975). Short-term memory processes and the amnesic syndrome. In D. Deutsch \& J.A. Deutsch (Eds.), Short-term memory (pp. 258-291). New York: Academic Press.
Kopelman, M.D. (1989). Remote and autobiographical memory, temporal context memory and frontal atrophy in Korsakoff and Alzheimer patients. Neuropsychologia, 27, 437-460.

Kopelman, M.D. (1993). The neuropsychology of remote memory. In F. Boller \& J. Grafman (Ed.), Handbook of neuropsychology (Vol. 8, pp. 215-238). Amsterdam: Elsevier.

Lucchelli, F., De Renzi, E., Perani, D., \& Fazio, F. (1994). Primary amnesia of insidious onset with subsequent stabilisation. Journal of Neurology, Neurosurgery and Psychiatry, 57, 1366-1370.

McClelland, J.L., McNaughton, B.L., \& O’Reilly, R.C. (1995). Why there are complementary learning systems in the hippocampus and neocortex: Insights from the successes and failures of connectionist models of learning and memory. Psychological Review, 102, 419-457.

Murre, J.M.J. (1996). TraceLink: A model of amnesia and consolidation of memory. Hippocampus, 6, 675-684.

Nelson, H.E. (1982). The National Adult Reading Test (NART): Test manual. Windsor, U.K.: NFER-Nelson.

Osterrieth, P.A. (1944). Le test de copie d'une figure complexe: Contribution à l'étude de la perception et de la mémoire [The Complex Figure Copy Test: Its contribution to the study of perception and memory]. Archives de Psychologie, 30, 206-356.

Parkin, A.J. (1982). Residual learning capability in organic amnesia. Cortex, 18, 417-440.

Patterson, K. \& Hodges, J.R. (1992). Deterioration of word-meaning: Implications for reading. Neuropsychologia, 30, 1025-1040.

Raven, J.C. (1962). Coloured Progressive Matrices Sets A, AB, B. London: H.K. Lewis.

Schacter, D.L. (1987). Implicit memory: History and current status. Journal of Experimental Psychology: Learning, Memory and Cognition, 13, 501-518.

Schwartz, M.F., Marin, O.S.M., \& Saffran, E.M. (1979). Dissociation of language function in dementia: A case study. Brain and Language, 7, 277-306.

Shimamura, A.P. \& Squire, L.R. (1987). A neuropsychological study of fact memory and source amnesia. Journal of Experimental Psychology: Learning, Memory and Cognition, 13, 464-473.

Snodgrass, J.G. \& Vanderwart, M. (1980). A standardised set of 260 pictures: Norms for name agreement, familiarity and visual complexity. Journal of Experimental Psychology: General, 6, 174-215.

Snowden, J.S., Goulding, P.J., \& Neary, D. (1989). Semantic dementia: A form of circumscribed cerebral atrophy. Behavioural Neurology, 2, 167-182.

Snowden, J., Griffiths, H., \& Neary, D. (1994). Semantic dementia: Autobiographical contribution to preservation of meaning. Cognitive Neuropsychology, 11, 265-288.

Squire, L.R. \& Alvarez, P. (1995). Retrograde amnesia and memory consolidation: A neurobiological perspective. Current Opinion in Neurobiology, 5, 169-177.

Tanabe, H., Kazui, H., Ikeda, M., Hasikawa, K., Hasimoto, M., Yamada, N., \& Eguchi, Y. (1994). Slowly progressive amnesia without dementia. Neuropathology, 14, 105-114.

Tulving, E. (1972). Episodic and semantic memory. In E. Tulving \& W. Donaldson (Eds.), Organization of memory (pp. 381403). New York: Academic Press.

Tulving, E. (1983). Elements of episodic memory. New York: Oxford University Press.

Verfaellie, M., Reiss, L., \& Roth, H.L. (1995). Knowledge of new English vocabulary in amnesia: Examination of premorbidly acquired semantic memory. Journal of the International Neuropsychology Society, 1, 443-453. 
Warrington, E.K. (1975). The selective impairment of semantic memory. Quarterly Journal of Experimental Psychology, 27, 635-657.

Warrington, E. K. (1984). Recognition Memory Test. Windsor, U.K.: NFER-Nelson.

Wechsler, D. (1987). Wechsler Memory Scale-Revised manual. San Antonio, TX: The Psychological Corporation.
Weintraub, S. \& Mesulam, M.M. (1993). Four neuropsychological profiles in dementia. In F. Boller \& J. Grafman (Eds.), Handbook of neuropsychology (Vol. 8, pp. 253-282). Amsterdam: Elsevier Science.

Zola-Morgan, S., Cohen, N.J., \& Squire, L.R. (1983). Recall of remote episodic memory in amnesia. Neuropsychologia, 21, 487-500. 